\title{
Comparison of Different Experimental Methods for the Assessment of the Room's Acoustics ${ }^{1}$
}

\author{
Marina Dana Ţopa ${ }^{a}$, Norbert Toma ${ }^{a}$, Botond Sandor Kirei ${ }^{a}$, Ioana Homana ${ }^{a}$, \\ Marius Neag $a$, and Gilbert De Mey ${ }^{b}$ \\ ${ }^{a}$ Basis of Electronics Department, Faculty of Electronics, Telecommunications and Information Technology, \\ Technical University of Cluj-Napoca, Cluj-Napoca, Romania \\ ${ }^{b}$ Department of Electronics and Information Systems, Faculty of Engineering, Ghent University, Ghent, Belgium \\ e-mail: Marina.Topa@bel.utcluj.ro \\ Received August 10, 2010
}

\begin{abstract}
The paper presents the acoustics analysis of three different enclosed spaces. These spaces (rooms) have different geometrical shapes and sizes and serve for different purposes. The early decay time, reverberation time, clarity and center time are evaluated with Dirac, WinMLS, Aurora and Caracad software using simple, low-cost equipment. The listed acoustic parameters were determined using linear sine sweep and impulsive sources. Comparisons between experimental measurements, simulations and analytic results were done. The room impulse response measurement proved to be the most reliable method to evaluate the properties of different rooms even if the measurements are perturbed by non-idealities of the low-cost equipment.
\end{abstract}

DOI: $10.1134 / \mathrm{S} 1063771011020229$

\section{INTRODUCTION}

In an enclosed space the source sound meets the walls, ceiling and floor, giving birth to several reflections [1-3]. The reflections overlap the original sound, causing distortion. The sensation added by the reverberation phenomenon can be evaluated subjectively as pleasant (spatial sensation for music listeners) or annoying (unintelligibility of speech). Thus qualifying and quantifying the acoustic properties of enclosed spaces is the effort of common interest of the acoustic research societies. Although the main measurement procedures and acoustic parameters are defined in international standards, there are still discussions in the literature. Special attention is dedicated to the room impulse response (RIR) measurement (ISO 3382) because other parameters are extracted from the RIR 5-7. In [5], Farina provides a comprehensive overview of RIR measurement techniques with four different acoustic sources namely: impulsive sources, maximum length sources (MLS), linear sine sweep and exponential sine sweep. Using an impulsive source is the simplest method to register the RIR. This cheap solution provides fairly good measurements requiring only an acoustic impulse source (balloon, pistol or firecracker), pressure microphone, signal conditioner and a data acquisition system. The system using MLS consists of a computer, which generates the MLS signal (being a pseudorandom signal), this signal is passed through an audio amplifier, a loudspeaker. Measurement errors are caused by the non-idealities

\footnotetext{
${ }^{1}$ The article is published in the original.
}

of the amplifier and loudspeaker, thus the accuracy of the RIR is affected. A similar system is used for the time delay spectrometry (TPS), which uses a linear sine sweep signal. Holters concluded in [7] that the TPS allows the measurement of "relatively short impulse responses." The usage of the exponential sine sweep signal is also advised by Holters. The latter method presents better results regarding the signal to noise ratio (the RIR is considered to be the wanted signal) then the TPS.

Another topic we intend to touch in the introduction are the acoustic parameters. Myriad of acoustic parameters were defined: strength factor, clarity factors, center time, early decay time, reverberation time, time between incident and first reflection, bass ratio, inter-correlation index, definition etc. [9, 10]. These parameters are not independent ones. Based on this idea Cedra et al. presents a factor analysis approach for a dozen of enclosures located in the Spanish city Valencia [11]. Their conclusion is that a set of orthogonal acoustic parameter is enough to establish the properties of enclosed spaces. Earlier efforts for the determination of the significant acoustic parameters for Italian opera houses can be found in [12] identifying a series of elements that affects the measurement of the RIR.

Our previous research about the analysis and improvement of a room's acoustics using both software and hardware tools is presented in [13]. First a model of the room is proposed taking into account the 
comparison of simulation and experimental results. Next improvements in the architecture are suggested and then the optimal placement of loudspeakers and listeners is provided.

\subsection{Evaluated Acoustic Parameters}

The acoustic parameter evaluation is based upon the measurement of the RIR $h(t)$ and the computation of the energy decay curve (EDC) [4]. EDC computes the energy remaining in the room's impulse response after the time $t$ :

$$
\operatorname{EDC}(t)=\int_{t}^{\infty} h^{2}(\tau) d \tau .
$$

- The reverberation time T60 is the basic indicator of acoustical behavior and is the time required for the EDC to decay to $-60 \mathrm{~dB}$. If $V$ is the volume of the room, $S$ its surface and $\alpha$ the absorption coefficient of the walls, floor and ceiling, then T60 can be estimated according to the formulas of Sabine, Eyring and Kuttruff respectively 1,2 :

$$
\begin{gathered}
T_{60}=\frac{0.164 V}{\alpha S} ; \quad T_{60}=\frac{0.161 V}{-S \ln (1-\alpha)} ; \\
T_{60}=\frac{0.16 V}{S[-\ln (1-\alpha)]+\Delta+4 m V} ;
\end{gathered}
$$

where $\Delta$ is the mean reflection coefficient and $m$ the absorption coefficient of the air.

- The early decay time (EDT) is defined as the reverberation time from the decay range between 0 and $-10 \mathrm{~dB}$ on the EDC [3]. The EDT is strongly influenced by early reflections thus depends on the measuring position and the room's geometry. The associated subjective sensation of the reverberation is strongly dependent to EDT.

- Clarity $C_{t e}$ and definition $D_{t e^{*}}$. Clarity is the logarithmic ratio of an impulse response's energy before time $t_{e}$ and the energy after $t_{e}$, where $t_{e}$ equals 50 or $80 \mathrm{~ms}$. Definition is similar to clarity, but expressed in $\%$ instead of $\mathrm{dB}$.

$$
C_{t_{e}}=10 \log \frac{\int_{0}^{t_{e}} h^{2}(t) d t}{\int_{t_{e}}^{\infty} h^{2}(t) d t}[d B] ; D_{t_{e}}=\frac{\int_{0}^{t_{e}} h^{2}(t) d t}{\int_{t_{e}}^{\infty} h^{2}(t) d t} \times 100[\%] .
$$

The clarity is the ratio, expressed in $\mathrm{dB}$, between the "useful energy" which is received in the first $50(80) \mathrm{ms}$ and the "detrimental energy" which is received afterwards [3].
Both clarity and definition are measures of the distinctness and clarity of speech and music [2].

- The center time $\left(\boldsymbol{T}_{c}\right)$ corresponds to the gravity center of the impulse response energy:

$$
T_{c}=\frac{\int_{0}^{\infty} t h^{2}(t) d t}{\int_{0}^{\infty} h^{2}(t) d t}[s] .
$$

\subsection{Measurement Setup and Software}

The acoustic parameters estimation consists of the following steps:

- Measure the room impulse response (RIR) — in our experiments the following equipment was used:

- two loudspeakers (one for low frequencies and the other for medium and high frequencies) - for rendering the excitation;

—a portable cardiode microphone (dB VH 210) for acquiring the response;

-balloons inflated at the same pressure (50 $\mathrm{mm} \mathrm{Hg}$ );

-firecrackers;

—an omnidirectional microphone (PCB 130D20).

- Estimation of the acoustic parameters-the measurements processing was done with various softwares as Dirac, WinMLS, Aurora or CARACAD.

\subsection{Evaluated Enclosures}

The evaluated enclosures are used for different purposes. The first room is a multiuse midsize school hall situated in the buildings of the "Iuliu Maniu" Technical College in Carei (Romania), with the volume of approximately $1952 \mathrm{~m}^{3}$. The room does not contain any furniture. The second evaluated enclosure is St. Maria Catholic Church located in Satu-Mare (Romania). Beside the regular religious services the church gives home for smaller events and concerts. The volume of this space is $4011 \mathrm{~m}^{3}$ and its furniture consists of wooden benches. The last room is an auditorium of the Technical University of Cluj Napoca (Romania), with similar size and purpose as the first school hall, but the walls are covered with absorbing materials as wood and plexiglass.

This paper presents different experimental methods for an objective assessment of the room's acoustics, using simple low-cost equipment and available software; a comparison regarding the best method is provided. 


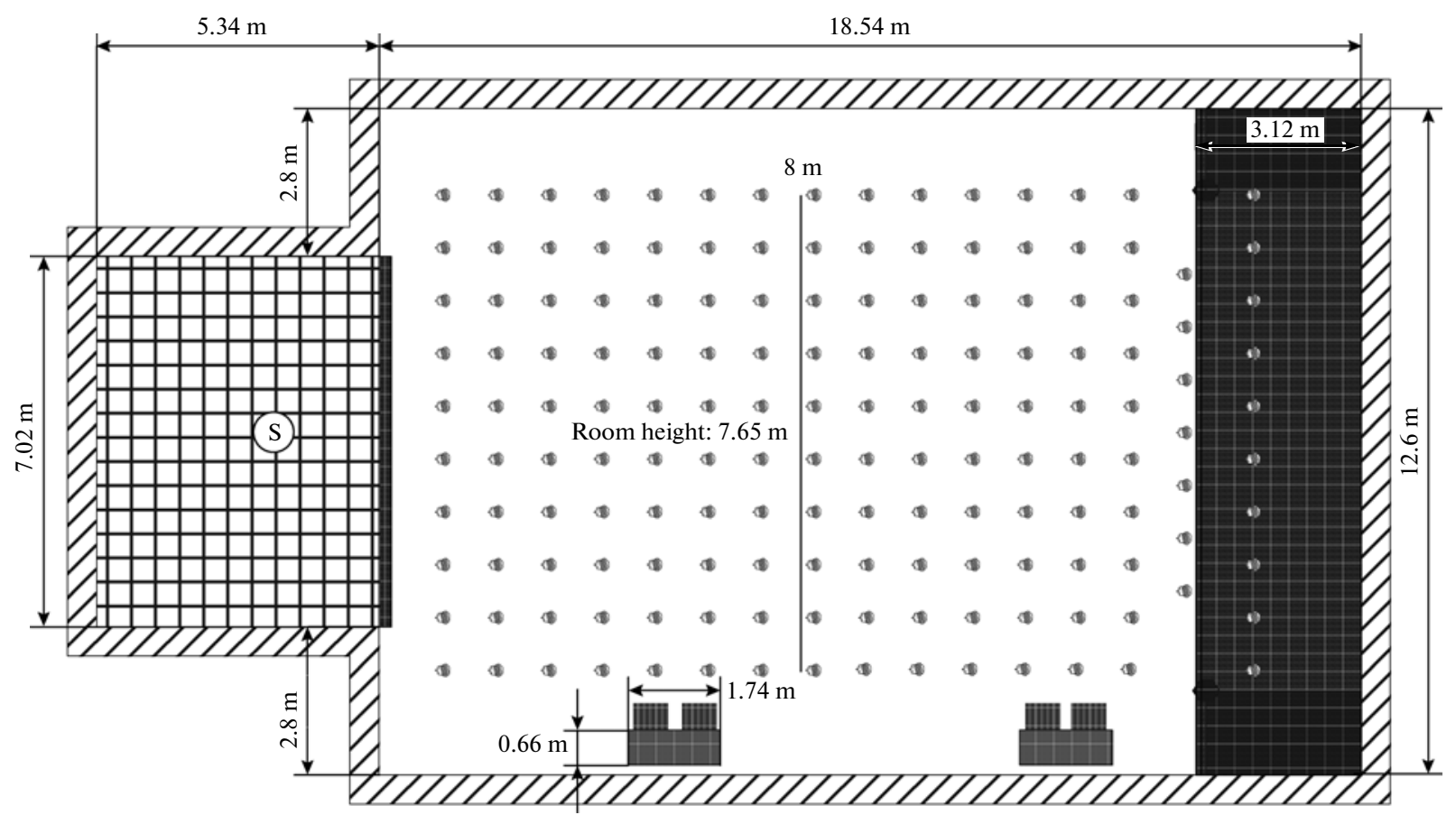

Fig. 1. The plan of the school hall.

The paper is organized as follows: Section 2 presents the estimation of the acoustic parameters in a school-hall, Section 3 in a church and Section 4 in an auditorium. Each room was analyzed in a different way and its conclusions regarding the best method were used in the next analysis to improve its quality. The last section presents the final conclusions altogether with the comments related to the acoustic properties of the analyzed rooms.

\section{THE EVALUATION OF ACOUSTIC PARAMETERS USING SWEEP SINE AND IMPULSIVE SIGNALS}

The first examined room was the school-hall depicted in Fig. 1. The measurements were carried out in 164 locations in the room: these locations are noted with grey points in Fig. 1. It is worth mentioning that the school-hall was measured without chairs. The source $S$ is pointed out with a white circle and there are two ovens placed near the wall (black and grey rectangles). In every location the measurement was performed 5 times and its mean value was taken into consideration. Two types of excitations were used: the impulsive signal obtained using balloons and the software generated sweep sine signal. The processing of the measurements was done in Dirac or WinMLS softwares. In the case of the reverberation time estimates were provided also by the acoustics simulator CARACAD, according to (2).
The estimation of the reverberation time with different methods is presented in Fig. 2a. One can see that there are not important differences between the values obtained by measurements in the Dirac or WinMLS software or for different excitation signals, because T60 does not depend on the excitation source. There are significant errors between measurements and simulations at frequencies below $500 \mathrm{~Hz}$. The errors in CARACAD were due to rough approximations as: impossibility of modeling the complex form of all the objects in the room, very rude approximation of some objects shape (circles were approximated by hexagons) as well as in component materials in the room.

The mean values of the EDT are plotted in Fig. 2b. The corresponding mean values of the clarity, according to (3), are depicted in Fig. 2c. Figures $3 a$ and $3 b$ presents the frequency dependence of the clarity in the points belonging to the line placed at $8 \mathrm{~m}$ distance from the stage (see the 8th line in Fig. 1). If for the sweep signal large variations are obtained at high frequencies, for impulsive excitation they occur at low frequencies. Similar spread was observed for all acoustical parameters. These errors are due to different signal-to-noise ratios (SNR); for the sweep sine the SNR has low values at medium and high frequencies $(<40 \mathrm{~dB})$. At low frequencies the mean value of the SNR is above $40 \mathrm{~dB}$ with high deviations. The impulsive signal has low values for the SNR at $125 \mathrm{~Hz}$, but for other frequencies the SNR is above $40 \mathrm{~dB}$ with 

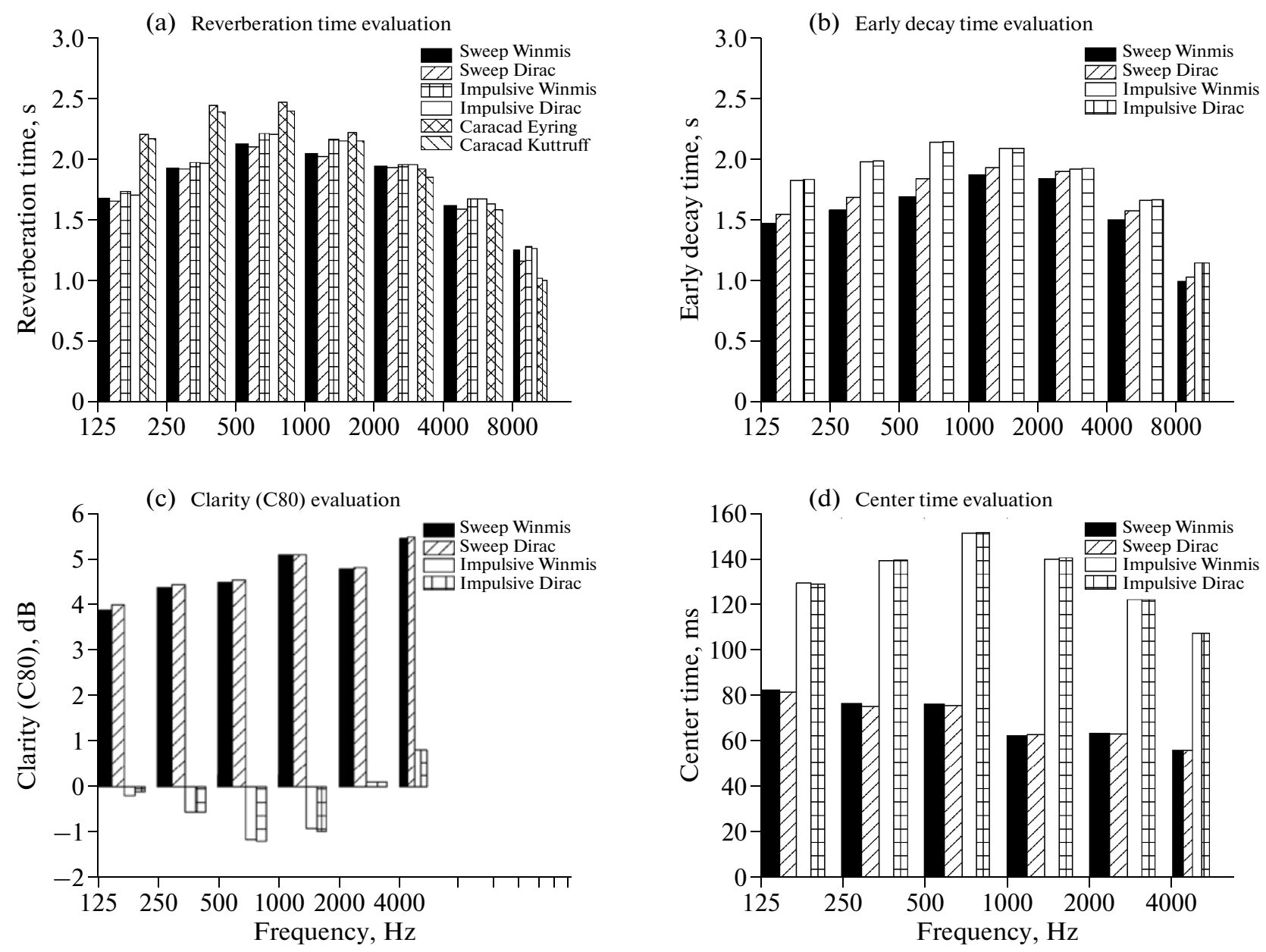

Fig. 2. School-hall characteristics, measured and simulated mean values for: (a) the reverberation time; (b) early decay time; (c) clarity; (d) center time.
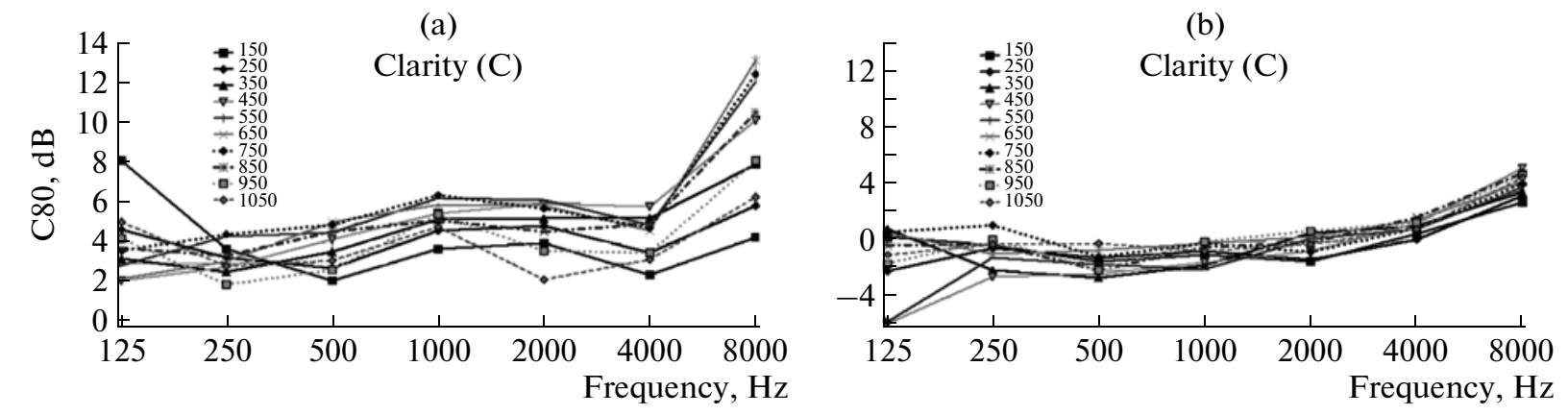

Fig. 3. School-hall characteristics: (a) the clarity on the 8th line for sweep sine signal excitation. (b) The clarity on the 8th line for impulsive signal excitation.

smaller deviations from the mean value than the sweep sine excitation (Fig. 4).

The estimation of the central time defined in (4) is represented in the Fig. 2d. There are huge differences between the values obtained with different excitations. Larger variations were obtained when exciting the room with a sweep sine signal.
A general remark about the evaluation of the acoustic parameters can be done: if the reverberation and EDTs do not depend considerably on the excitation source type, the listener's position or the room's shape, the other parameters are very sensitive to them. The acoustic parameters are not correctly measured for the sweep sine signal, due to the large variations of 

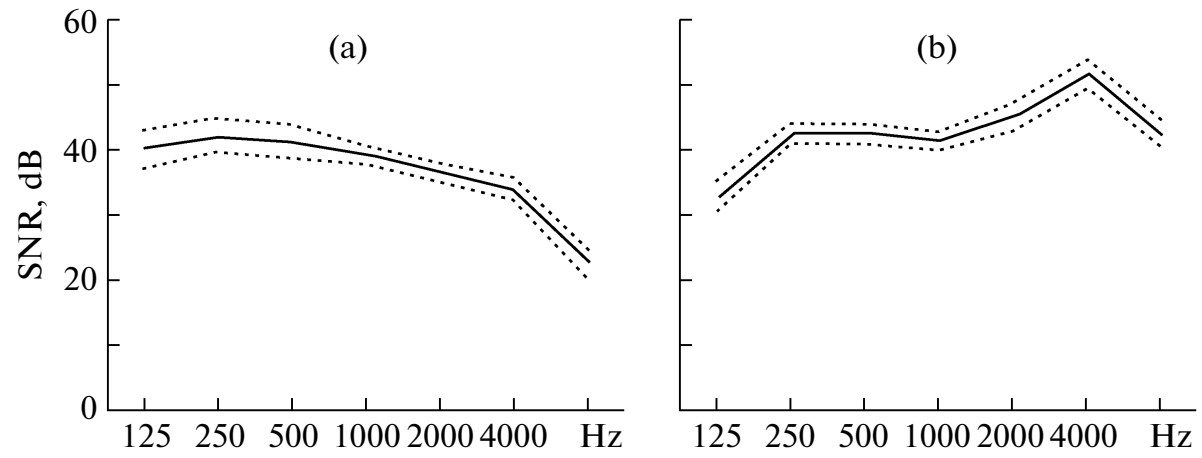

Fig. 4. Global error from mean SNR values for the excitation being (a) sweep sine signal; (b) impulsive signal.

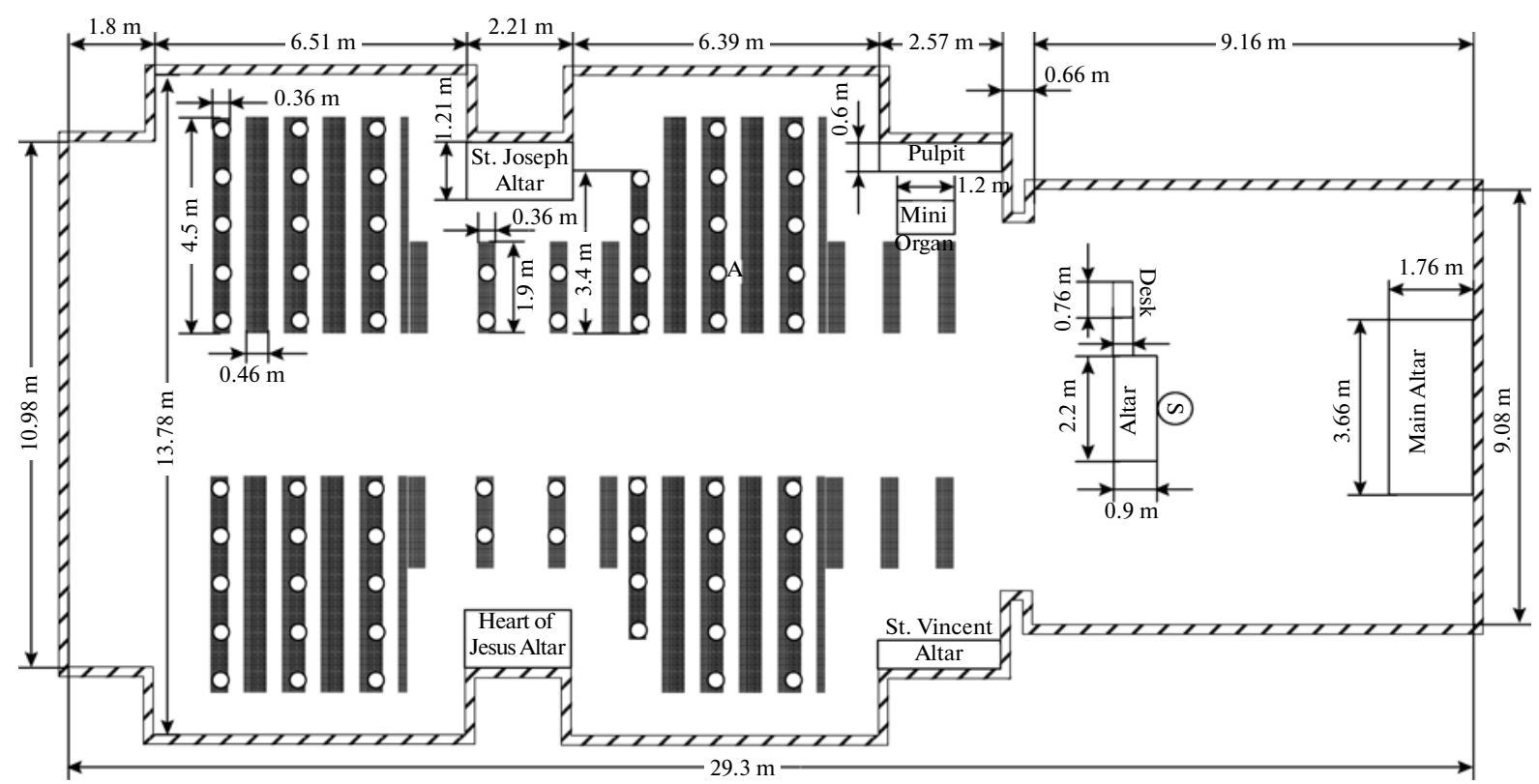

Fig. 5. Plan of the St. Maria Catholic Church.

the SNR. The impulsive source leads to better measurements. As for the processing software, both WinMLS and Dirac softwares give similar results.

\section{THE EVALUATION OF ACOUSTIC PARAMETERS USING IMPULSIVE SIGNALS GENERATED BY BALLOONS AND FIRECRACKERS}

The second analyzed room was a church; its plan is presented in Fig. 5. Taking into consideration the previous experiments, the measurements were performed only with impulsive signals; they were generated by balloons or firecrackers; the source $\mathrm{S}$ is indicated by the white large circle. The measurements points are noted in Fig. 5 by white small circles and their number is 66. The processing of the measurements was done with WinMLS, Dirac and Aurora.
The main of these experiments was to see which of the low-cost devices are more reliable: balloons or firecrackers. The first step was to make 5 measurements in the same spot with the two available impulse generators. The chosen spot for RIR measurement was the 4th seat in the second line, noted with $\mathrm{A}$ in Fig. 5. To check the repeatability of the measurements, we kept the same conditions: exact positions of source and microphone, same pressure of the balloons, etc.

The values of the clarity obtained with balloons measurement are depicted in Fig. 6a and the ones with firecrackers in Fig. 6b; the spread of the values obtained with balloons is larger than that of the firecrackers.

The repeatability was checked also after doing all the measurements in the 66 locations in the church. 

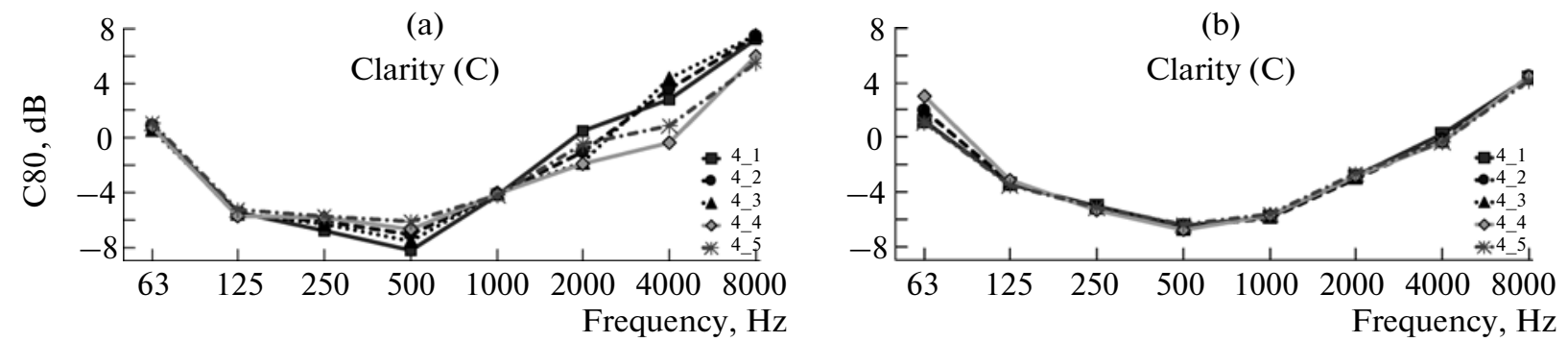

Fig. 6. Clarity values for measurements done in the point $A$ of the church with impulsive signals generated by (a) balloons; (b) firecrackers.
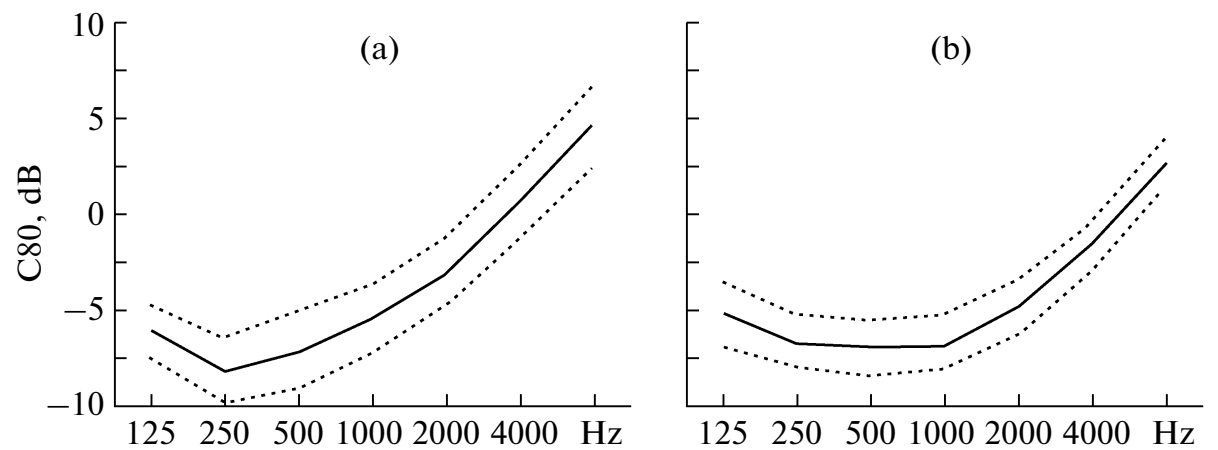

Fig. 7. Global error from clarity mean values for measurements in all the points of the St. Maria Catholic Church with impulsive signals generated by (a) balloons; (b) firecrackers.
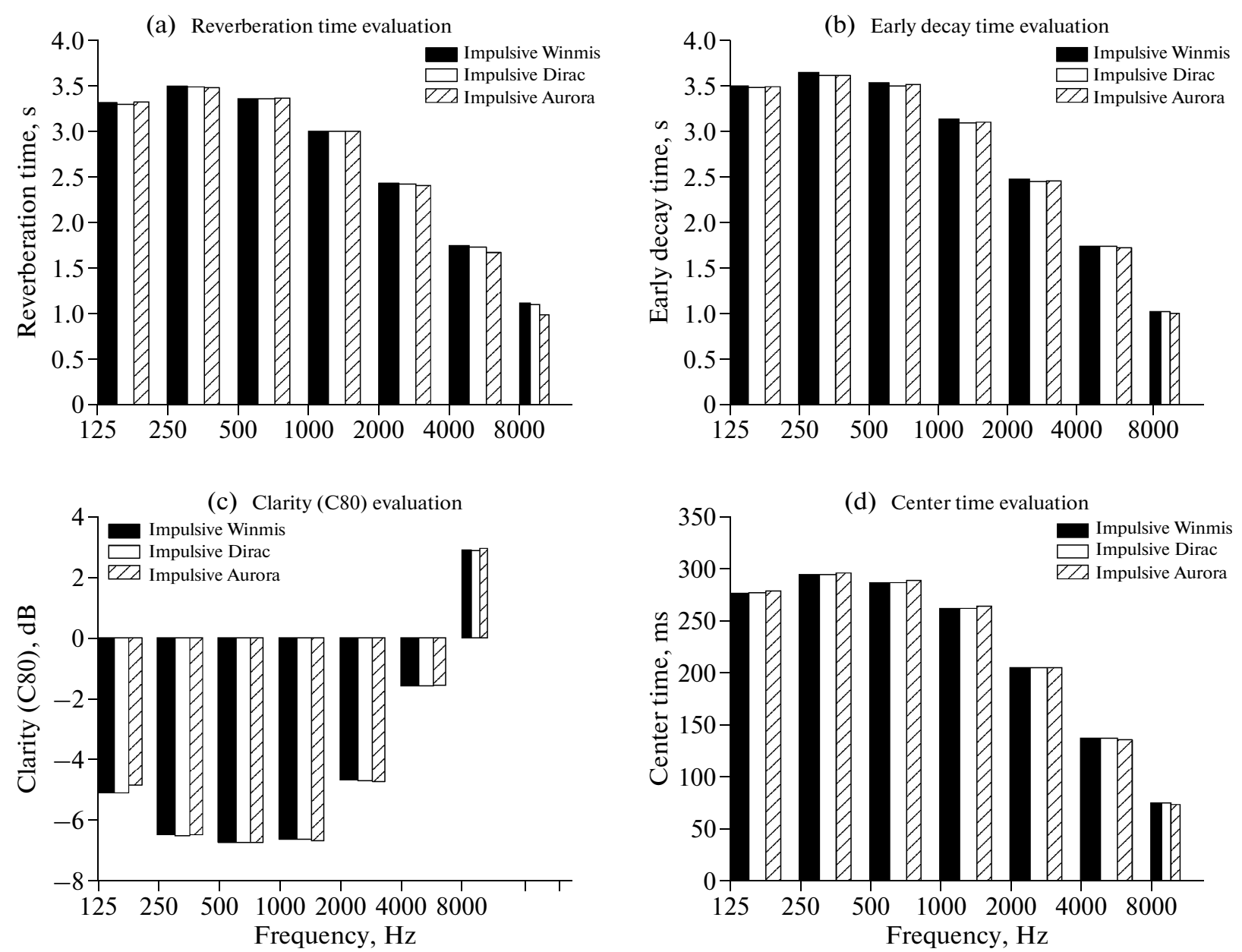

Fig. 8. St. Maria Catholic Church characteristics, measured mean values for (a) the reverberation time; (b) early decay time; (c) clarity; (d) center time. 
(a)

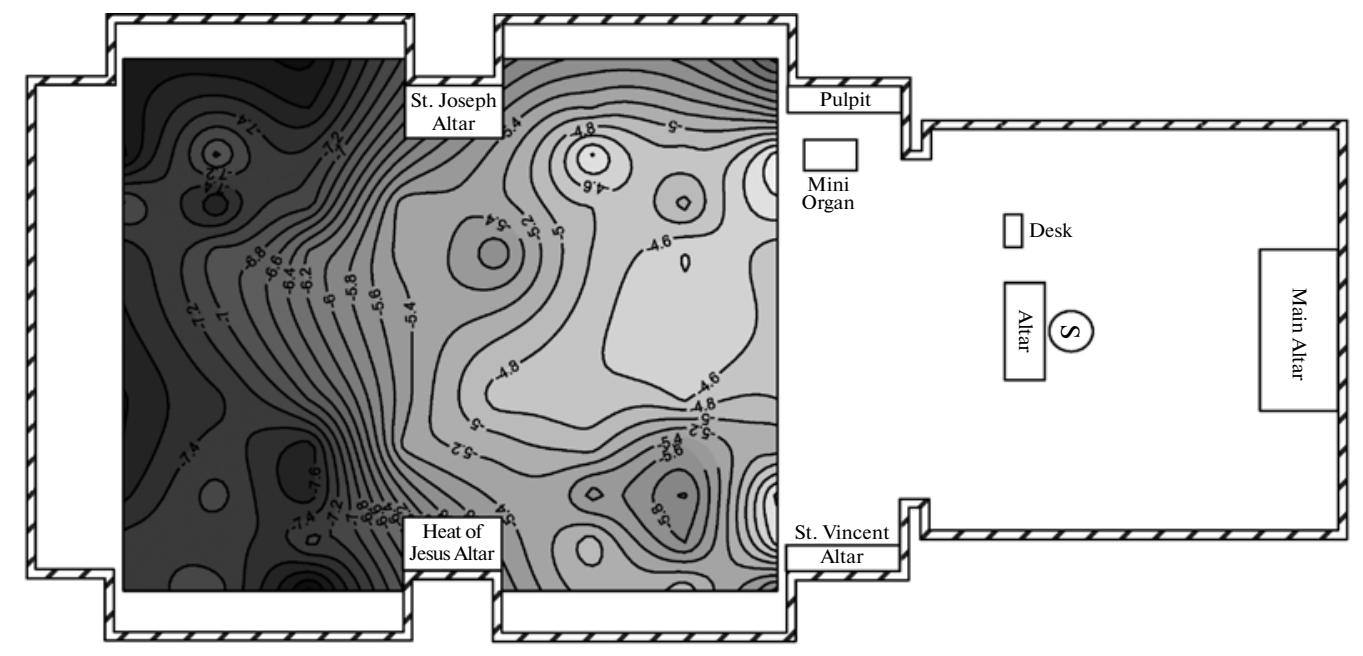

(b)

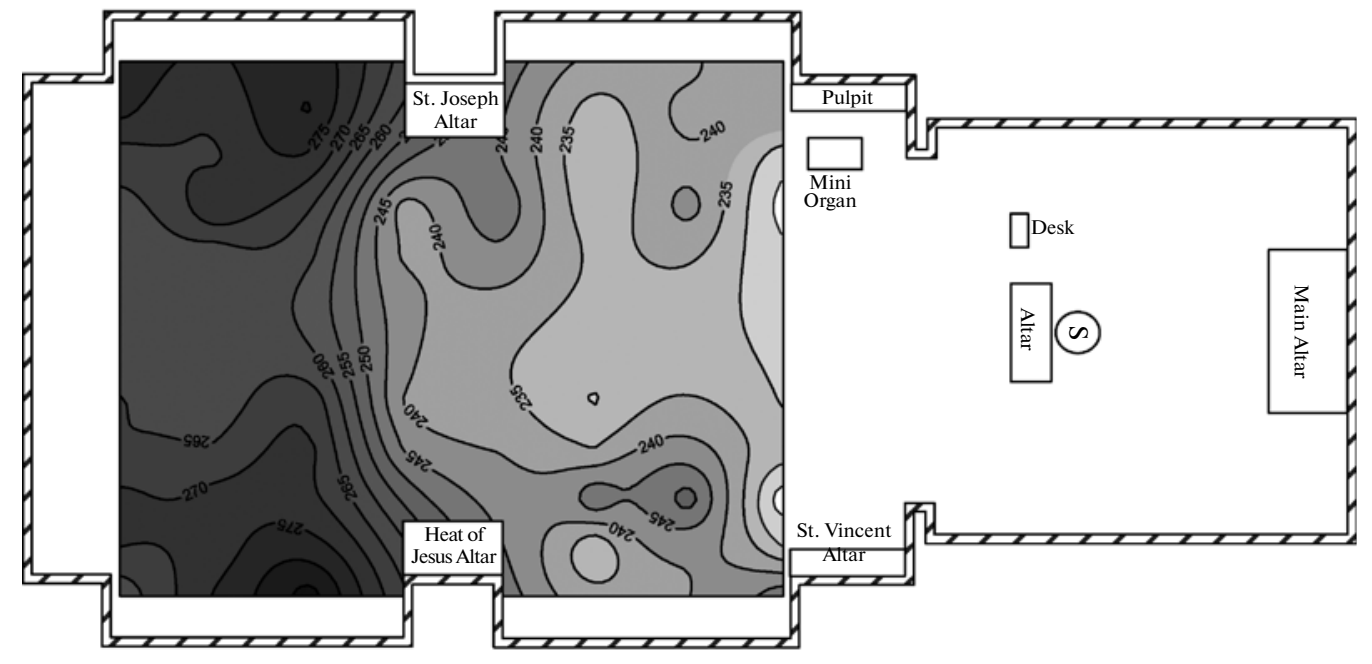

Fig. 9. Maps of St. Maria Catholic Church: (a) map of the clarity; (b) map of the center time.

Figures $7 \mathrm{a}$ and $7 \mathrm{~b}$ show the error from the mean value of the clarity for all the measurements points. The curves point out that deviation of results is less for firecrackers than for balloons.

Examining Figs. $7 \mathrm{a}$ and $7 \mathrm{~b}$ the following conclusion is drawn: the firecrackers are more reliable than balloons, their repeatability being better than that of the balloons. In the followings we considered only the measurements obtained with firecrackers and we estimate the acoustic parameters using the WinMLS, Dirac and Aurora softwares. Figures 8a to 8d show the mean values of the reverberation time, EDT, clarity and center time. The obtained values do not depend on the used software.

To have a better insight into the acoustic properties of the church maps of the acoustic parameters sensitive to the listener's position were plotted (Figs. 9a and 9b). If for the clarity map (Fig. 9a), the white color corresponds to $-4 \mathrm{~dB}$, the black is for $-8.4 \mathrm{~dB}$. Knowing that negative values mean reverberant spaces and positive values denote dead spaces and that the optimal clarity values depend on type of music, the church is very good for listening to organ, concerts, but not to conferences. In Fig. 9b white is associated to $220 \mathrm{~ms}$ and black to $290 \mathrm{~ms}$; for music listening the center time must have large values ( $>70 \mathrm{~ms})$.

\section{THE EVALUATION OF ACOUSTIC PARAMETERS IN AN AUDITORIUM}

The third analysis example was an auditorium; its plan is presented in Fig. 10. In this case the impulsive source was generated by firecrackers and estimation of some acoustic parameters was also provided by 


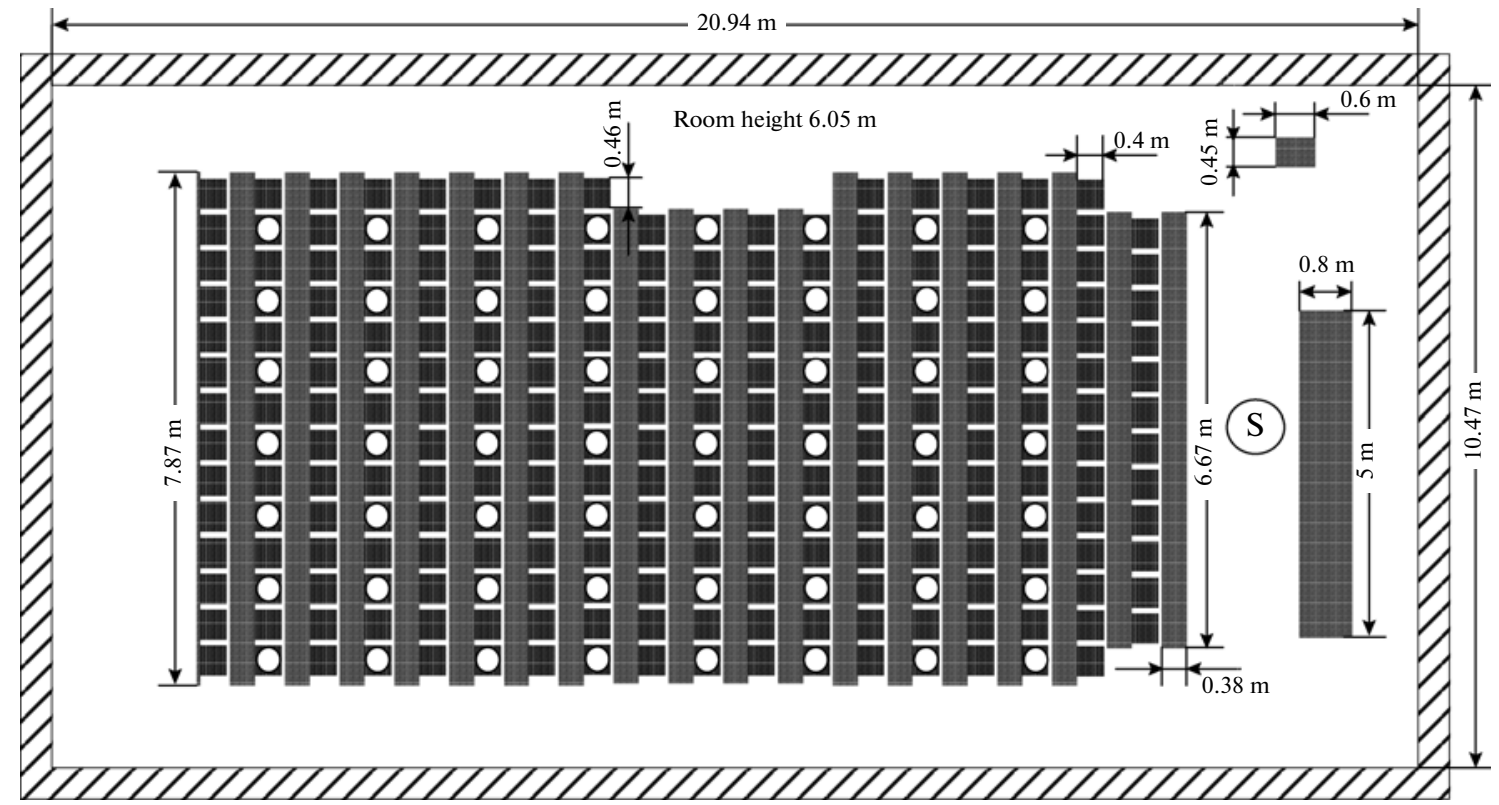

Fig. 10. The plan of the auditorium.

(a) Reverberation time evaluation

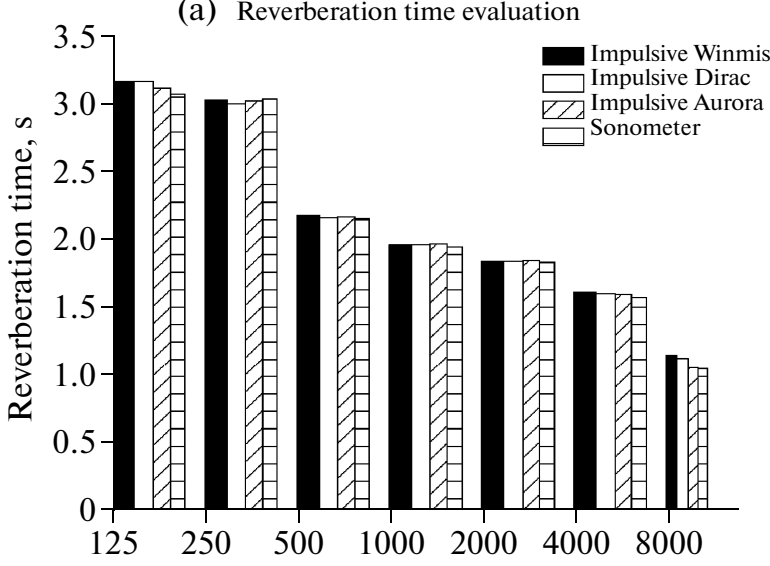

(c) Clarity (C80) evaluation

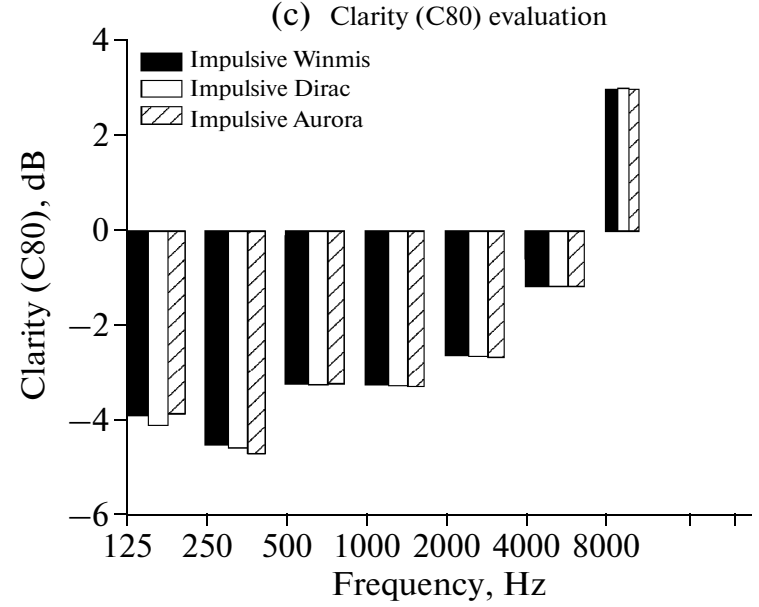

(b) Early decay time evaluation

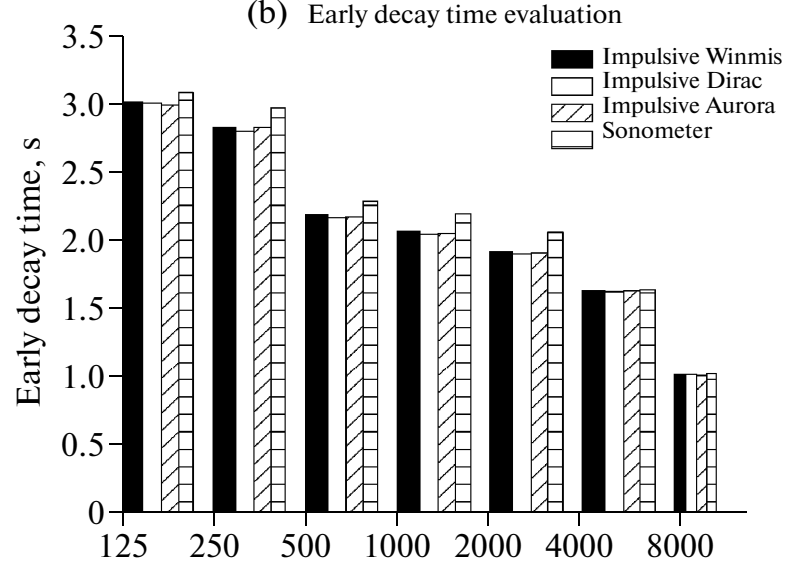

(d) Center time evaluation

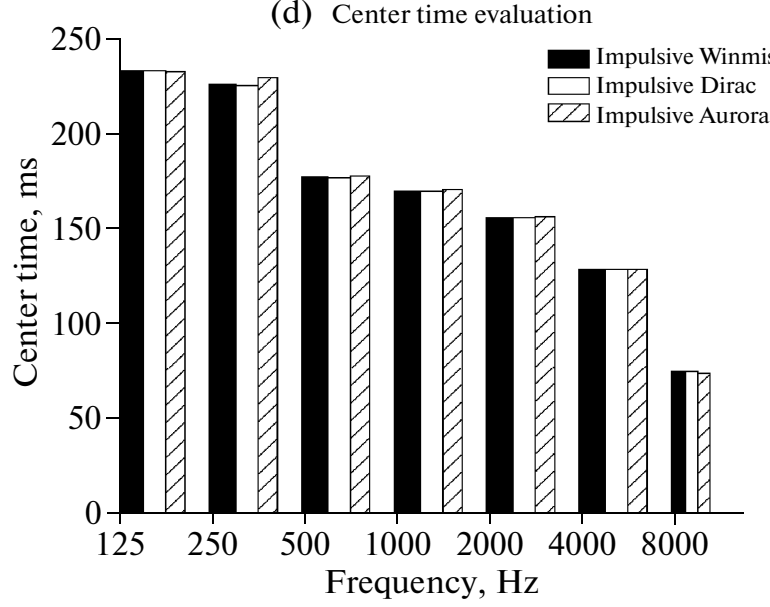

Fig. 11. Auditorium characteristics, measured mean values for (a) the reverberation time; (b) early decay time; (c) clarity; (d) center time. 
a sonometer. The source $\mathrm{S}$ was placed in the front part of the auditorium (see white large circle in Fig. 10) and the measurements were done in the spots pointed out by small white circles in Fig. 10. The data processing was done with WinMLS, Dirac and Aurora.

Figures 11a and $11 \mathrm{~b}$ show the mean values for the reverberation and EDTs. The values obtained by measurements are similar to the ones provided by the sonometer, so the validity of the measurements with firecrackers followed by software processing is confirmed. Figures $11 \mathrm{c}$ to $11 \mathrm{~d}$ present the mean values of clarity and center time in the auditorium.

\section{CONCLUSIONS}

The paper is focused on the estimation of the acoustic parameters of a room. The evaluation is based upon the measurement of the room impulse response. The best simple and low-cost method to excite the room was found to be the generation of impulsive signals using firecrackers. Software processing of the measurements lead to the same values whatever software was chosen (WinMLS, Dirac or Aurora). At the same time the obtained values for the reverberation and EDT are similar with those provided by a sonometer.

If the reverberation and EDTs do not depend considerably on the excitation source type, the listener position or the room's shape, the other parameters (clarity, center time) are very sensitive and maps of their values can help significantly in the improvement of the room acoustics. It is worth mentioning that the school-hall was measured without chairs and the other two with seats. Neither of the rooms is populated.

In what is the reverberation time concerned, all the analyzed rooms are in the range for concert-halls $(>1.6 \mathrm{~s})$. The typical range of clarity $\mathrm{C} 80$ is from about -5 to $+3 \mathrm{~dB}[1]$. The church is a very reverberant space when not populated, so it is suited for organ concerts. In the church intelligibility is poor $(\mathrm{C} 80<-4 \mathrm{~dB})$. From the clarity point of view the School-Hall and the auditorium is suited for symphonic concerts. The center time is a much more stable acoustical parameter than clarity and definition. High speech intelligibility is indicated by low values $(<80 \mathrm{~ms})$, so all the analyzed rooms could be used for musical events.

\section{ACKNOWLEDGMENTS}

This work was supported by the Romanian National University Research Council under Grant ID 1057 entitled "2.5D Modeling of Sound Propagation in Rooms and Improvement of Room Acoustical Properties using Digital Implementations.”

\section{REFERENCES}

1. H. Kuttruff, Room Acoustics (Spon Press, London, UK, 2009), p. 109.

2. F. A. Everest, Master Handbook of Acoustics, 4th ed. (McGraw-Hill, Two Penn Plaza, New York, 2001), p. 54.

3. M. Kahrs and K. Brandenburg, Applications of Digital Signal Processing to Audio and Acoustics (Kluwer Acad., Norwell, MA, 1998), pp. 89-100.

4. ISO 3382, Acoustics-Measurement of the Reverberation Time of Rooms with Reference to Other Acoustical Parameters (Intern. Organization for Standardization, 1997).

5. A. Farina, in Proceedings of the 23rd Nordic Sound Symposium Training and Information Seminar for Audio People, 27-30 Sept. 2007, pp. 1-31, http://pcfarina.eng.unipr. it/Public/Papers/238-NordicSound2007.pdf.

6. R. Stewart and M. Sandler, in Proceedings of the 10th Intern. Conference on Digital Audio Effects (DAFx-07), Bordeaux, France, Sept. 10-15, 2007, pp. 59-62, http://dafx.labri.fr/main/dafx07-proc.pdf.

7. M. Holters, T. Corbach, and U. Zólzer, in Proceedings of the 12th Intern. Conference on Digital Audio Effects (DAFx-09), Como, Italy, Sept. 1-4, 2009, pp. 1-5, http://dafx09.como.polimi.it/proceedings/data/ DAFx09_Proceedings.pdf.

8. M. R. Schroeder, Acoust. Phys. 49, 97 (2003).

9. A. Pompei, M. A. Sumbatyan, and N. F. Todorov, Acous. Phys. 55, 821 (2009).

10. M. Yu. Lannie and V. N. Sukhov, Acoust. Phys. 47, 623 (2001).

11. S. Cedra, A. Gimenez, J. Romero, R. Cibrian, and J. L. Miralles, Appl. Acoust. 70, 97 (2009).

12. P. Fausti and A. Farina, J. Sound Vibrat. 232, 213 (2000).

13. N. Toma, M. D. Țopa, I. Muresan, B. S. Kirei, M. Neag, and A. Fazakas, Acta Tehn. Napocensis: Electron. Telecommun. 50 (2) (2009). 